\title{
FORUM
}

\section{Wege aus der Verfassungskrise - die EU nach den gescheiterten Referenden in Frankreich und den Niederlanden}

\author{
Mathias Jopp und Gesa-S. Kuhle*
}

Das negative Votum der Franzosen und der Niederländer zum Vertrag über eine Verfassung für Europa (VVE) - verstärkt durch das gescheiterte Gipfeltreffen der Staats- und Regierungschefs am 16./17. Juni 2005 - konfrontiert die Europäische Union mit einer Krise, deren Ausmaß noch nicht absehbar ist. Die hohe Beteiligung bei den Referenden in Frankreich und den Niederlanden und die große Stimmendifferenz zwischen den Lagern der Befürworter und Gegner sowie insbesondere die Tatsache, dass ausgerechnet die Bürger zweier Gründerstaaten die europäische Verfassung derart deutlich abgelehnt haben, zwingen die europäische Politik, innezuhalten und nach Auswegen aus der Krise zu suchen. Was sind die hauptsächlichen Ursachen für die Ablehnung des VVE und welche Schlüsse sind daraus für die Europapolitik zu ziehen?

\section{Ursachen für die Ablehnung des Verfassungsvertrages}

Bei der Ablehnung des Verfassungsvertrages spielten in erster Linie innenpolitische Verwerfungen, in zweiter Linie allgemeine europapolitische Gründe und zum geringsten Anteil direkte Kritikpunkte an der Verfassung eine Rolle. ${ }^{1}$ Dies ist einerseits ein trauriges Resultat mit Blick auf die eigentlich in Frage stehende Sache, andererseits ein Hoffnungsschimmer, den Verfassungsvertrag nicht als verloren anzusehen - vor allem, nachdem das Ratifizierungsverfahren bereits in zehn Mitgliedstaaten abgeschlossen wurde, in denen 52 Prozent der EU-Bevölkerung leben.

Die hohe Bedeutung der innenpolitischen Gründe zeigt sich daran, dass beispielsweise in Frankreich überwältigende 55 Prozent der Befragten, die mit Nein gestimmt hatten, die soziale Situation als Begründung für ihr Negativvotum zum VVE nannten. ${ }^{2}$ Die sich kontinuierlich verschlechternden Wirtschaftsdaten und soziale Sparmaßnahmen führten dort wie auch in den Niederlanden zu einer Ablehnung der Politik der Regierungsparteien und damit ihres Werbens um Zustimmung zur Europäischen Verfassung. Obwohl die nationalen Regierungs- und die meisten Oppositionsparteien in ihrer offiziellen Linie pro-europäisch argumentierten, schlossen sich die französischen und niederländischen Wähler mehrheitlich den Argumenten der Rand- und Extremparteien sowie in Frankreich außerdem den Argumenten der innerparteilichen Opposition der Sozialisten und der politischen Bewegung ATTAC an. Das ,Denkzettel-Motiv ' gegenüber der jeweiligen Regierung war stark ausgeprägt ${ }^{3}$ und die Verdrossenheit über die nationale Politik bildete einen idealen Anknüpfungspunkt für populistische Bestrebungen all jener, die sich über eine Negativkampagne zur Europäischen Verfassung innenpolitisch zu profilieren versuchten.

* Dr. Mathias Jopp, Direktor des Instituts für Europäische Politik, Berlin. Gesa-S. Kuhle, M.E.S., wissenschaftliche Mitarbeiterin, Institut für Europäische Politik, Berlin.

1 Für eine detaillierte Darstellung der französischen Referendumsdebatte siehe den Beitrag von Joachim Schild in dieser Ausgabe.

2 Vgl. Umfrage des Instituts CSA: „Le vote au referendum sur le traité constitutionnel européen: Explication du vote et perspectives politiques“, Mai 2005. 
Die Argumente der Verfassungsgegner enthielten jedoch auch eindeutig allgemeine europapolitische Gründe. In Frankreich standen beispielsweise die Kritik an der neoliberalen Orientierung der europäischen Wirtschaftsintegration, die Auswirkungen der EU-Erweiterungspolitik - insbesondere die Aussicht auf einen möglichen EU-Beitritt der Türkei - und die Befürchtung eines nationalen Einfluss- und Identitätsverlustes in der größer gewordenen Europäischen Union im Vordergrund. Etliche der vorgebrachten Argumente waren verknüpft mit der Kritik am sozialen ,Fall out‘ von, überregulierter' Binnenmarktpolitik (wie etwa der Dienstleistungsrichtlinie) oder mit der Angst vor „Überfremdung“4 und einem Verlust des Arbeitsplatzes wegen des befürchteten Zuzugs billiger Arbeitskräfte aus neuen Mitgliedstaaten. In den Niederlanden konzentrierte sich der Protest zudem auch auf die dem Euro angelastete Teuerungsrate und die Position des Landes als größtem EU-Beitragszahler gemessen am Bruttonationaleinkommen (BNE) ${ }^{5}$, bei gleichzeitigen Bemühungen der Regierung Balkenende, den EU-Stabilitätspakt mittels eines nationalen Sparhaushalts einzuhalten.

Der geringste Teil der Gegenargumente der Nein-Kampagnen hatte tatsächlich Bezug zur Europäischen Verfassung. In Frankreich wurde beispielsweise kritisiert, dass der Verfassungsvertrag einer Verfassung im eigentlichen Sinne nicht genüge. Die Gegner lehnten insbesondere den Teil III des VVE ab, da seine Inhalte nicht denen einer Verfassung nach französischem Verständnis entsprächen. Der Oppositionspolitiker Laurent Fabius (Parti Socialist) oder die Basisbewegung ATTAC bezogen sich auf einzelne Artikel im VVE und lieferten hierzu teilweise völlig falsche Interpretationen; ${ }^{6}$ oder sie argumentierten sogar unseriös, da sie die Fortschritte gegenüber dem Vertrag von Nizza nicht darstellten. Dabei ging es um genau diese Entscheidung, die allerdings den wenigsten Teilnehmern an den Referenden in Frankreich und den Niederlanden klar war: für den Verfassungsvertrag und gegen den Vertrag von Nizza oder eben umgekehrt.

\section{Widersprüche und Gegensätze auf Seiten der Verfassungsgegner}

Eine Vielzahl der französischen ,Nein-Sager' hofft auf neue Verhandlungen für einen besseren Verfassungsvertrag. Es bleibt jedoch ein Rätsel, wie ein neuer Kompromiss aussehen kann, wenn alleine schon die Verfassungsgegner in den Niederlanden beispielsweise ganz andere wirtschaftspolitische Ziele in der Europapolitik verfolgen als die in Frankreich und sich darüber hinaus solche Auffassungsunterschiede in der EU-25 noch multiplizieren. Immerhin bleibt positiv festzustellen, dass 72 Prozent der französischen Wähler eine Fortset-

3 In Frankreich nannten zwischen 30 und 40 Prozent der befragten Nein-Wähler „Unzufriedenheit mit der aktuellen politischen Situation“ als Motivation für ihr Votum zum VVE, in den Niederlanden waren dies mit nur 8 Prozent bedeutend weniger, vgl. Umfragen von France 2 vom 29. Mai 2005, http://referendum-constitution-europeenne.france2.fr/11031227-fr.php (letzter Zugriff: 22.6.2005), und von Libération vom 29. Mai 2005, http:/ /www.louis-harris.fr/version_f/autres_s/Acrobat (letzter Zugriff: 22.6.2005) sowie Umfrage von Maurice de Hond vom 01. Juni 2005, erhältlich über www.peil.nl.

4 Seit den politisch motivierten Morden an dem Politiker Pim Fortuyn und dem Regisseur Theo Van Gogh reagieren die Niederländer äußerst sensibel auf Einwanderungs- und Integrationsfragen. Mit steigender Zahl der EU-Mitglieder befürchten sie eine Verschärfung der Integrationsproblematik in den Niederlanden.

5 Zwar wird zumeist Deutschland als größter so genannter, Nettozahler' bezeichnet, doch bezogen auf die Wirtschaftskraft der Mitgliedstaaten sind die Niederlande der stärkste Nettozahler: Im Jahr 2003 zahlten die Niederlande nach Berechnung der Einnahmen und Ausgaben 0,43 Prozent ihres BNE an den EU-Haushalt, Deutschland und Schweden jeweils nur 0,36 Prozent. Frankreich zahlte im selben Zeitraum nur 0,12 Prozent seines BNE an die EU.

6 In der französischen Referendumsdebatte kursierte beispielsweise das Gerücht, die Festschreibung der Gleichheit zwischen Mann und Frau fehle im VVE oder er führe das Abtreibungsverbot wieder ein. Diese Argumente sind eindeutig falsch. Der VVE schreibt einen allgemein verbindlichen Wertekatalog für die EU fest, der unter anderem die Gleichstellung von Frau und Mann regelt (Art. I-3 VVE) und keine national festgelegten Rechte, wie beispielsweise das Recht auf Abtreibung, einschränkt. 
zung der Europäischen Integration befürworten, ${ }^{7}$ auch wenn über deren Ziele und Inhalte keine einheitliche Auffassung besteht. Während etwa Fabius für mehr supranationale Elemente und den Abbau des Einstimmigkeitsprinzips in der Sozialgesetzgebung warb, blieben rechte Parteien (Front National und Souveränisten) ihrer europaskeptischen Grundlinie treu und forderten ,weniger Europa' und mehr intergouvernmentale Entscheidungsverfahren. Auch im Hinblick auf die Niederlande finden sich kaum Parallelitäten. Denn in Frankreich dominiert ein Europabild eines weniger deregulierten und eher protektionistisch sozial orientierten Europas mit durchaus weicherem Euro, in den Niederlanden steht das Bild eines Europas mit effizienterem Binnenmarkt bei fairem Wettbewerb und einem stabilitätsorientierten Euro im Vordergrund.

Die Ablehnungsgründe deuten also auf eine Gemengelage mit teils widersprüchlichen, sich überlagernden oder sogar ausschließenden Elementen hin. In einigen Bereichen hat sich die Ablehnung sogar selbst ad absurdum geführt: Französische Verfassungsgegner traten für Transparenz und mehr Demokratie in der Union ein und lehnten dennoch den Verfassungsvertrag ab, obwohl der VVE gerade auf diesen Gebieten Fortschritte gegenüber dem Vertrag von Nizza beinhaltet.

\section{Schlussfolgerungen für die EU}

Zunächst gilt es, Risiken bei Referenden zu reduzieren. Der überdeutliche Bezug zur nationalen politischen Arena und der feststellbare Argumentationswirrwarr in den öffentlichen Kampagnen gemahnen zur Vorsicht bei europapolitischen Volksbefragungen, besonders in Zeiten innenpolitischer Unzufriedenheit und aktueller Zukunftsängste. Europäische Primärrechtstexte sind zudem als völkerrechtliche Verträge Resultat schwieriger Kompromisse zwischen mittlerweile 25 Mitgliedstaaten. Für Nein-Kampagnen bieten sich zahlreiche Angriffsflächen, die mit dem Kompromisscharakter eines solchen Vertragswerkes zusammenhängen. Die Verfassungsgegner nutzen diese Situation, um mit radikalen, extremen und teilweise falschen Argumenten die Schwächen eines solchermaßen austarierten Kompromisspaketes anzuprangern und dies mit einer Abstrafung der Regierung zu verbinden, wie die Referendumsdebatten in Frankreich und den Niederlanden gezeigt haben. Referenden sollten deshalb nur sehr dosiert zum Einsatz kommen, und zwar dort, wo sie verfassungsmäßig vorgeschrieben sind oder wo sie Aussicht auf Überwindung einer parlamentarischen Niederlage bieten. Vor allem muss die Fragestellung bei einem Referendum sehr genau überlegt und der Sache nach angemessen formuliert werden, damit die Wahl zwischen Verfassung oder Nizza-Vertrag und auch die Konsequenzen des individuellen Votums für die Gemeinschaft der 25 Mitgliedstaaten sowie für das eigene Land erkennbar werden. Der gesamte Kontext für ein europapolitisches Referendum würde in ferner Zukunft erst dann anders aussehen, wenn die EU-Bürger die Möglichkeit hätten, einen Konvent zur Ausarbeitung einer Europäischen Verfassung zu wählen, der dann wiederum das Ergebnis seiner Arbeit in einem europaweiten, zentral ausgezählten und zeitgleich durchgeführten Referendum zur Abstimmung stellt. Zwar wäre der Ausgang eines solchen Referendums ebenfalls ungewiss, der Hauptunsicherheitsfaktor, die Bedeutung der nationalen Arena wäre jedoch relativiert. Die vom Europäischen Rat am 16./17. Juni 2005 erklärte Entzerrung des Ratifizierungsfahrplans muss genutzt werden, um deutlich auf die Sorgen und Ängste der europäischen Bürger zu reagieren und insgesamt eine pro-aktive Politik zur Problembewältigung zu betreiben. Dies könnte zunächst die Zurückhaltung kritischer Binnenmarktrichtlinien bedeuten, vor allem müsste aber eine intensivere Wirkungsanalyse und Subsidiaritätsü-

7 Vgl. IPSOS-Umfrage zum Referendum, 29. Mai 2005. 
berprüfung von europäischen Gesetzesvorhaben durch die Kommission ${ }^{8}$ vorgenommen werden, wobei die nationalen Parlamente schon jetzt besser einbezogen werden könnten ${ }^{9}$. Zudem ist ein behutsameres Vorgehen in der Erweiterungspolitik empfehlenswert. Letzteres kann jedoch nicht heißen, die auf der Liste stehenden Beitrittskandidaten vor den Kopf zu stoßen, vor allem nicht, wenn schon Beitrittsverträge geschlossen oder Verhandlungsversprechungen gemacht wurden. Dies wäre im Hinblick auf die Stabilisierungs- und Demokratisierungsziele kontraproduktiv. Generell muss aber die Linie in der Erweiterungspolitik klarer eingehalten werden: Die Kriterien für die Verhandlungsaufnahme und den Beitritt müssen erfüllt, die Aufnahmefähigkeit der Europäischen Union durch institutionelle und politikbereichsspezifische Reformen gewährleistet und die Einigung auf eine Finanzierung bis zum Jahre 2013 gesichert sein. Zudem müssen Alternativen zum Beitritt entwickelt und insbesondere die europäische Nachbarschaftspolitik verbessert und ausgebaut werden, um einerseits den Erweiterungsdruck von der Union zu nehmen und andererseits den reformorientierten Kräften in den Nachbarstaaten Unterstützung zu geben. ${ }^{10}$

Eine offensivere und pro-aktivere Antwort auf die Bürgersorgen bezüglich der Problemlösungsfähigkeit durch die Europäische Union muss sich auf eine rasche Einigung über das Finanzpaket konzentrieren und mittelfristig die europäischen Kernprobleme angehen: Arbeitslosigkeit und schwaches Wirtschaftswachstum. Da die Union im Bereich der Wirtschaftsund Beschäftigungspolitik äußerst dürftige oder gar keine Zuständigkeiten hat, sind die notwendigen Strukturreformen eine vorrangige Aufgabe der Mitgliedstaaten, die hier in der Verantwortung stehen. Die Union kann aber ergänzende Strategien zur Unterstützung und Stimulierung der Mitgliedstaaten anbieten. So ist nach der jetzigen Halbzeitwertbewertung die ,fußlahme“ Lissabonstrategie, die auch eine Europäische Beschäftigungsstrategie beinhaltet, zu überarbeiten und auf die wichtigsten Wachstumsfelder und neueren Ansätze in der Economic governance auszurichten. Auch könnte ergänzend an eine verbesserte Neuauflage der im Jahr 2000 ausgelaufenen „Initiative Wachstum und Beschäftigung“ zur Unterstützung der Gründung und Finanzierung kleinerer und mittlerer Unternehmen gedacht werden. Zudem muss die im November 2003 vom Europäischen Rat angenommene Europäische Wachstumsinitiative forciert umgesetzt werden. Dies ist sinnvoll, da sie auf eine Überprüfung der Ausgabenstruktur der Union hinsichtlich ihrer investiven Wirkungseffekte zielt. Hierbei geht es beispielsweise um eine intensive europäische Förderung von Forschung und Ausbildung, grenzüberschreitender Infrastruktur sowie von Verkehrsprojekten. Der langfristige Haushalt der Europäischen Union muss weniger auf strukturkonservierende und mehr auf zukunftsorientierte Aufgaben ausgerichtet werden.

Der Argumentations-Wirrwarr bei den Referenden in Frankreich und den Niederlanden weist auf die dringliche Aufgabe hin, besser und konstant über die Europäische Union aufzuklären und zu informieren (und nicht jetzt eine leicht ausufernde Debatte über Sinn und Zweck der EU-Integration zu führen und damit das Rad neu zu erfinden, das bereits 26 Monate lang im Konvent und während der Regierungskonferenz ausgiebig und mit vorzeigbaren Ergebnissen gedreht wurde). Erforderlich sind europapolitische Öffentlichkeitsstrategien, die die Menschen in ihren Alltagswahrnehmungen erreichen. Hierfür ist ein radikales Umdenken erforderlich. Vor allem muss eine solide europapolitische Öffentlichkeitsarbeit auch von den nationalen Regierungen durchgeführt und wesentlich intensiviert werden. Hier

8 Vgl. Art. 4 EGV.

9 Vgl. Protokoll 9 EGV sowie Protokoll 2 VVE, insbes. Art. 6.

10 Vgl. Barbara Lippert: Die Türkei als Sonderfall und Wendepunkt der klassischen EU-Erweiterungspolitik, in: integration 2/05, $28 \mathrm{Jg} .$, S. 119-135. 
gibt es in allen Mitgliedstaaten einen großen Nachholbedarf. Die Bürger müssen nachvollziehen können, dass sich ihre Regierung für die Union und den Verfassungsvertrag engagiert und welche Gründe dafür sprechen. Die Inhalte des VVE müssen besser vermittelt und die mit ihm verbundenen Fortschritte gegenüber dem Vertrag von Nizza hinsichtlich Demokratie und Transparenz deutlicher herausgestellt werden. Da es sich als naiver Irrglaube herausgestellt hat, dass eine vorhandene pro-europäische Haltung (wie in Frankreich) ausreicht, um EU-Vertragsreformen plebiszitär ratifizieren zu können, empfiehlt es sich grundsätzlich, vor Ratifizierungsprozessen eine Analyse der Sorgen der Bürger vorzusehen, um sehr frühzeitig und mit gezielten Informationen auf diese eingehen zu können. Generell sind in der Öffentlichkeitsarbeit die Stärken und die Schwächen europäischer Projekte offen darzustellen. Neue Schritte in der Vertiefung der EU-Integration oder der Erweiterung bedürfen intensiver Begründung. Die Frage von Alternativen müsste erörtert und deren politische und ökonomische Kosten im Vergleich zum eingeschlagenen Integrationspfad dargestellt werden. Vor allem muss die Rolle der Europäischen Union bei der Bewältigung der Globalisierungsherausforderungen in Kombination mit notwendigen einzelstaatlichen Strukturreformen aufgezeigt und mit den fatalen Konsequenzen eines Rückzugs in nationale , Schutz- und Trutzburgen" verglichen werden.

\section{Eine Strategie zur Verwirklichung des Verfassungsvertrags}

Die Verlängerung des Ratifizierungsprozesses muss als Diskussions- und Reflexionsphase genutzt werden, um die Verwirklichung des Europäischen Verfassungsvertrags zu ermöglichen. Denn er bietet für die entscheidenden Probleme der Gestaltung des politischen Systems der erweiterten Union mit Blick auf die europäische Handlungsfähigkeit und Effizienz die zurzeit und auf lange Sicht bestmöglichen Lösungen an. In der Folge der Juni-Erklärung des Europäischen Rates wird nunmehr jede Regierung der 13 Mitgliedstaaten, die noch nicht den Verfassungsvertrag ratifiziert haben, entscheiden, wie und wann sie die Situation für geeignet hält, die Ratifizierung einzuleiten beziehungsweise durchzuführen. In der Zwischenzeit könnte zumindest schon eine, weiche" Konstitutionalisierung unterhalb der Vertragsänderungsschwelle beginnen, das heißt ein pragmatisches Vorwegnehmen von Aspekten des Verfassungsvertrags, soweit dies auf der Basis des Nizza-Vertrags etwa im Rahmen der Außen- und Sicherheitspolitik, der Innen- und Justizpolitik und durch interinstitutionelle Vereinbarungen zwischen den europäischen Institutionen - wenn auch nur in begrenztem Umfang - machbar ist. Im ersten Halbjahr 2006 werden dann die Staatsund Regierungschefs unter österreichischer Präsidentschaft den Fortgang des Ratifizierungsprozesses vereinbaren. Dies kann die Beibehaltung des jetzigen Kurses oder eine weitere Verschiebung im Ratifizierungskalender bedeuten. Über einen Abbruch des Ratifizierungsverfahrens sollte aber nicht entschieden werden, ohne zunächst eine hochrangige Reflexionsgruppe einzusetzen, die unter Berücksichtigung aller gemachten Erfahrungen mit dem Ratifizierungsprozess Optionen und Strategien zur Annahme des Verfassungsvertrages erarbeitet und in einem Bericht dem Europäischen Rat Ende 2006 oder Anfang 2007 vorlegt. Der deutschen Ratspräsidentschaft würde dann im ersten Halbjahr 2007 wesentliche Verantwortung für die weitere Weichenstellung zukommen.

Der Beitrag ist im Rahmen des Forschungsprojekts „Von der geschriebenen zur gelebten Verfassung “ entstanden, das gemeinsam vom Institut für Europäische Politik (IEP) und der ASKO-EUROPA-STIFTUNG durchgeführt wird. 